\title{
Design of Intelligent PD Controller for Water Supply in Healthcare Systems
}

\author{
Mohan Debarchan Mohanty ${ }^{1}$, Mihir Narayan Mohanty ${ }^{2 *}$ \\ ${ }^{I}$ Instrumentation \& Electronics Engineering, College of Engineering, Bhubaneswar India \\ ${ }^{2}$ Electronics \& Communication Engineering, ITER, \\ Siksha 'O' Anusandhan Deemed to be University, Bhubaneswar, India \\ Imohan.debarchan97@gmail.com, ${ }^{2}$ mihir.n.mohanty@gmail.com
}

\begin{abstract}
The necessity of clean environment is the major aspect in this modern age. This maintains a healthy environment. Also several trials have made with multidisciplinary researchers for development of healthy environment. However, it is even important to facilitate the unhealthy people in the healthcares. Some extent technology has an important role to support it. In this paper, a case study of water supply in the modern health care units is analyzed. This work describes the application of rule based Fuzzy Logic for control operation in water supply section. To refine fuzzy rules' initial approximate set automatically, a self-organizing fuzzy controller has been used. The quality factor is increased by applying the PD-type fuzzy controller. To make the system robust, the controller has been designed with Fuzzy Logic rules. The simulation results confirm the advantages and demonstrate for better dynamic behavior and performance, as well as perfect control with no overshoot. For low energy consumption, either the energy input is decreased or the efficiency of the mechanical transmission and processes has been increased. Performance of these new controllers has been verified through simulation using MATLAB.
\end{abstract}

Index Terms-Modern Health Care; PD Controller; Fuzzy Controller; Intelligent Controller; Robustness.

\section{INTRODUCTION}

$\mathrm{I}$ $\mathrm{N}$ THE modern society, health care units are to be taken care sufficiently. Many e-health care units are already developed still the improvement is required minutely for this development of these units. Health care system is very large as it has many aspects such as patient entry, patient consultation, test and diagnosis. Also the requirements of physician with specialization along with the testing laboratories are to be improved for smart systems. Some of the works have been proposed earlier for above mentioned components [1] [4].

Similarly, the patients admitted in the hospitals have many requirements for healthy environmental condition. One such aspect is considered in this work is water supply in the toilets of the wards and room along with drinking water. An intelligent approach is considered with the help of Fuzzy logic (FL) to control the water flow to the tank and from this tank to the toilets and drinking water reservoir.

To control a steam engine and boiler combination, the Mamdani fuzzy inference system has been explored using linguistic rules [5] [6]. In this, the can be described using ifthen rules corresponding to the human language. Since no mathematical model is essential to design a FL controller, hence it maintains its robustness. Ease of application of FL makes it popular in industry. Nevertheless, knowledge database or expert experience is desired in deciding the rules or the membership functions (MF).

\section{Materials and Methods}

The proposed scheme aims to enhance the control performance water supply in the modern health care units. As compared to traditional controllers, the FL controllers possess the following advantages: they can be developed easily, can be customized readily in natural language terms and have very wider range of operating conditions. Practical systems are often complex and time variant in nature with delays or nonlinearities, and are often associated with improperly defined dynamics. Efforts are being made to provide easy and simple control algorithms by industries and researchers to accommodate increasing complexity in the processes/systems [7] [8].

Conventional control algorithms relying on linear system need to be linear zed before applied to systems, although the performance is not guaranteed. Nonlinear controllers can accommodate nonlinearities however; the lack of a general structure creates difficulty in their designing [9]. Thus, linear or nonlinear control algorithm solutions are mostly developed using precise mathematical system models. Difficulties in describing these systems using traditional mathematical relations happen to provide unsatisfactory design models or solutions [10]. This is a motivating factor to used for system design which can employ approximate reasoning to resemble the human decision process [11].

The FL Controllers do not depend on any mathematical model unlike conventional linear or nonlinear control theory to solve problems under vague and uncertain environments [12] [13]. This has made its use successfully and practically in a number of applications that include robotics and insurance [14] [17]. FL has been proposed by Zadeh in 1965 [11]. FL provides a specific artificial intelligence (AI) level to the traditional proportional and derivative (PD) controllers. Thus, Fuzzy PD controllers possess self-tuning 
ability as well as on-line adaptation to time varying, nonlinear, and uncertain systems.

The use of FL in the control systems better dynamic response, rejects disturbance, allows low parameter sensitive variation and removes external influences. The FL control algorithm tends to be robust, simple and efficient.

Experiments of the proposed control system have shown remarkable tracking performance, and demonstrate the usefulness and validity of the hybrid fuzzy controller convincingly with high-performance under parameter variations and load uncertainties. The performance has been satisfactory for most of the reference tracks.

Sometimes, the control system using microcontroller consumes more memory and power. Hence, it is advisable to operate the control system in MATLAB environment in a personal computer (PC) and by maintaining a duplex link between the robot and the personal computer. In this case, the microcontroller controls these wheels and at the same time need to be designed so as to receive the serial data via UART port. A function need to be simulated in MATLAB that can communicate with the four different codes and the robot using its serial port. These four codes are termed as move left, move forward, move right and stop. Fig.1 shows the design overview block diagram.

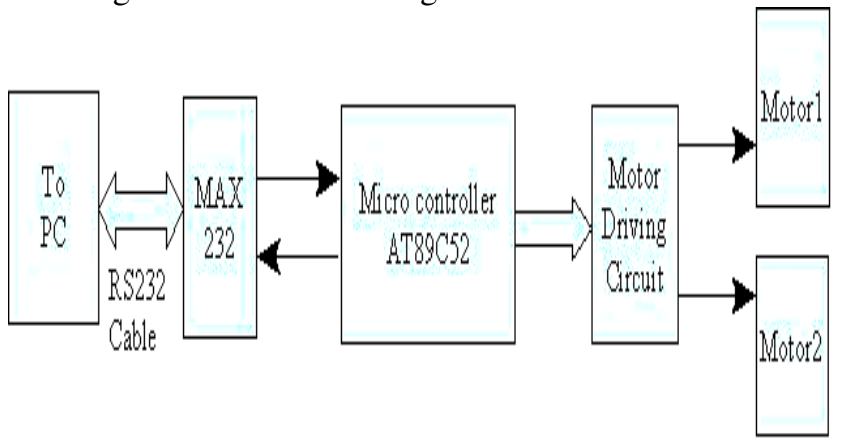

Fig.1. Block diagram of Design Overview

Though the RS-232 standard serial ports that have been used mostly on PC due to high baud rates, it cannot guarantee a favorable working connection. For example, for musical instrument control a special-purpose protocols named MIDI uses serial data rates instead of the above series.

\section{a) Fuzzy Controller}

Among the available AI techniques, the applications of fuzzy controllers have received attention. FL uses membership functions (MF)having values in the range of ' 0 ' and ' 1 ' to solve uncertainties or ambiguous problems. Because of uncertainties in the changing operating conditions of a power system, FL controller has found its potential application to consider uncertainties for system approximation. This is true the dependencies between the input control and output variables are not accurately specified. FL has been chosen here as non-linear controller because it is believed that deriving linguistic rule control action is a general design approach to avoid non-linear mathematical model complexity. Based on this ground here fuzzy logic is chosen to implement PD controller.
Fuzzy controllers require no design procedure, because they are rule based and can handle non-linearity. They do not require input and output relationship as a mathematical model. Same control scheme can be used for many problems. They can consider uncertainties and approximation derive control action from linguistic rules, easy to maintain and understand by untrained end users.

Fuzzy based controls are described as computing results with words rather than numbers i.e., with sentences not by equations. It includes linguistic rules in the form of IFTHEN format. The fuzzy controllers thus make the complex mathematics of the system very simple. The fuzzy IF-THEN rules use several variables while applying rule conditions or conclusion. The controllers need both changes in error and accumulation error as inputs. In this type of controller, a set of simple linguistic rules are evaluated. The rule development requires a clear understanding of the control process and system to be controlled although, mathematical system model is not essential. The inference method has been Mamdani while defuzzification accomplished using the centroid or center of area method.

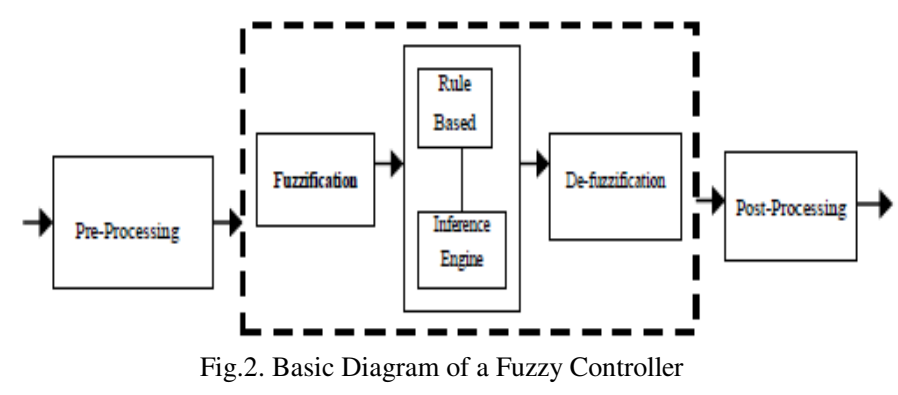

Fuzzy Based Controller

In PID controller design use of fuzzy rules generates few unwanted design issues. It follows a three step design approach in this work to design the PID control. These are:

(i) Begin with a PD controller.

(ii) Insert an equivalent with respect to the linear FL controller.

(iii) Gradually, make it non-linear.

(i) Specify the system input, states and output variables.

(ii) For each variable, partition the universe of discourse into different fuzzy subsets.

(iii) Assigning a linguistic label to each subsets.

(iv) Allot a FM to each of the fuzzy subsets.

(v) Determine the inputs and outputs fuzzy relationship to form the rule base system.

(vi) For the input and output variables, select an appropriate scaling factor so as to normalize them.

(vii) Fuzzily the inputs.

(viii) In order to infer the output contributed from each rule, apply fuzzy approximate reasoning.

(ix) Aggregate the fuzzy outputs that has been recommended by every rule.

(x) To develop the crisp output, apply defuzzification.

Fig. 3 shows the Fuzzy control block diagram 


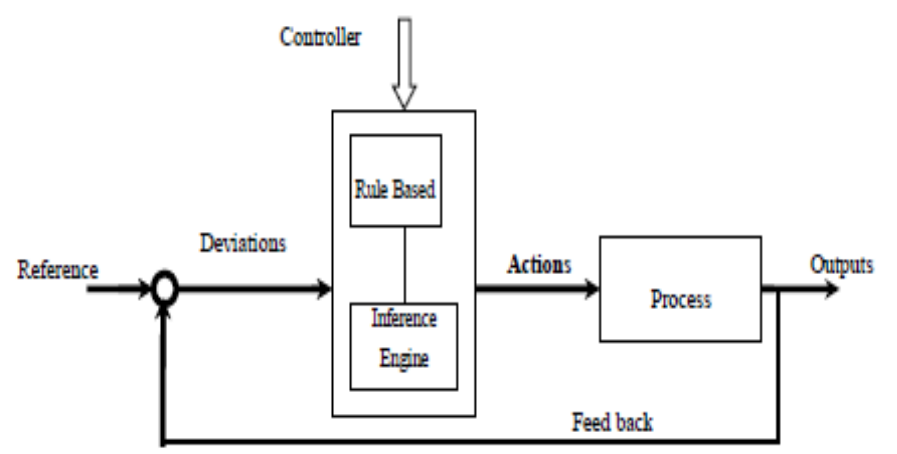

Fig.3. A Fuzzy Controller Block Diagram

\section{RESULT}

The experimental results may indicate the following observations:

1. Feasibility of a fuzzy based control algorithm Implementation on a micro controller platform.

2. The fuzzy controller can regulate the output voltage of boost or buck configurations to the specified value without undammed oscillations even in case of variation in input voltage or load.

3. The FL controller does not depend on the mathematical model but on a structure's linguistic explanation.

4. FL control schemes help to control dc-dc converter with cheap DSPs.

5. The design of the present controller is based on past experience to design the MFs and various controls.

6 . The contribution made in the present work shows that the FL control of PMDC motor can be designed at a low cost

7. Both the Buck and Boost converters can be controlled by making use of the same FL control algorithm, which will show the flexibility of the control scheme.

It is essential for inference rules to be modified during the tests on the equipment by reducing number of rules or to add complementary rules. The inference rule choice is made by taking in account the regulator implementation mod using a program or the equipment. The installation tests may be replaced with PC simulation in case the control system depends on the process model. Fig.4 shows the Fuzzy inference system editor menu.

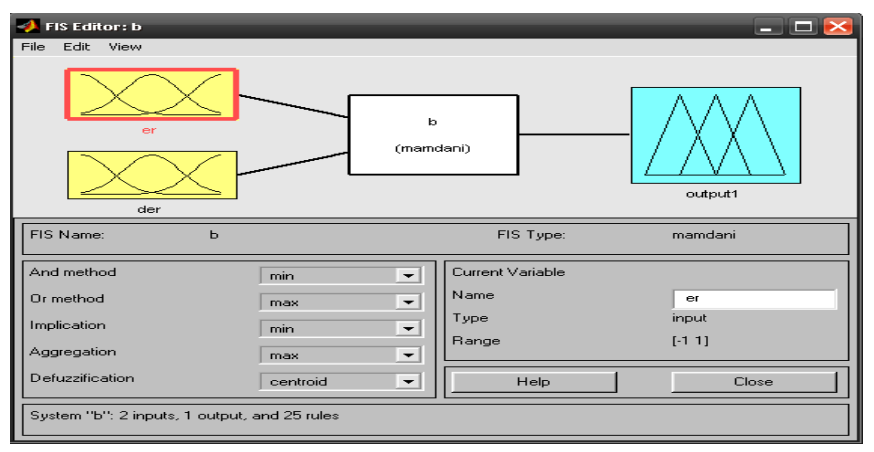

Fig.4. The editor of fuzzy inference system.
A FL controller makes due arrangement for the conversion of the linguistic control strategy into an automatic control strategy. In this case, the fuzzy rules are formulated based on knowledge database or expert experience.

The FL controller input variables are the angular velocity error $e(t)$ and its variation $\mathrm{d} e(t)$ whereas the output variable is the control. The linguistic variables that has been used are indicated as \{-veBig, -veSmall, Zero, +veSmall, +veBig\} corresponding to negative big, negative small, zero or Z, positive small and positive big respectively. The MF of the FL controller has been shown in Fig. 5 using Mamdani fuzzy inference and the corresponding fuzzy rules have been provided in Table 1.

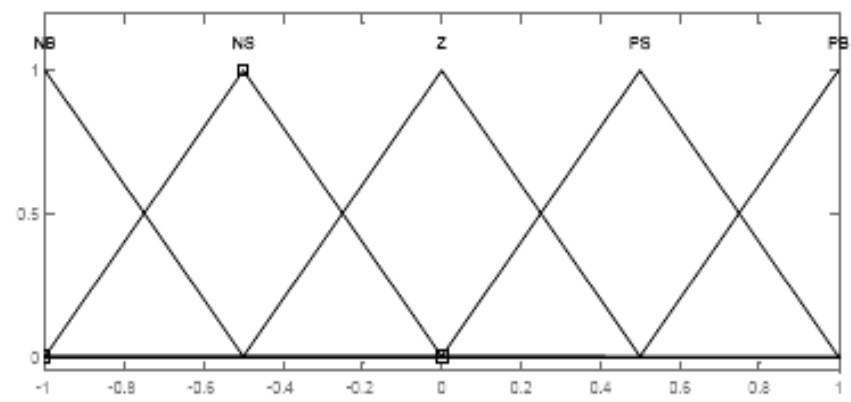

Fig.5. Membership function of Fuzzy logic controller

\begin{tabular}{|c|c|c|c|c|c|}
\hline $\begin{array}{c}\text { Error } \\
\text { Error } \\
\text { coefficient }\end{array}$ & $\begin{array}{l}+ \text { +ve } \\
\text { Big }\end{array}$ & $\begin{array}{l}+\mathrm{ve} \\
\mathrm{Big}\end{array}$ & $\mathrm{Z}$ & $\begin{array}{c}\text {-ve } \\
\text { Smal } \\
1\end{array}$ & $\begin{array}{l}\text {-ve } \\
\text { Big }\end{array}$ \\
\hline PB & $\begin{array}{c}- \\
\text { veB } \\
\text { ig }\end{array}$ & $\begin{array}{c}- \\
\text { veB } \\
\text { ig }\end{array}$ & $\begin{array}{c}\text {-ve } \\
\text { Smal } \\
1\end{array}$ & $\begin{array}{c}\text {-ve } \\
\text { Smal } \\
1\end{array}$ & $\mathrm{Z}$ \\
\hline PS & $\begin{array}{l}\text {-ve } \\
\text { Big }\end{array}$ & $\begin{array}{c}- \\
\text { veS } \\
\text { mall }\end{array}$ & $\begin{array}{c}\text {-ve } \\
\text { Smal } \\
1\end{array}$ & $\mathrm{Z}$ & $\begin{array}{c}\text { +ve } \\
\text { Small }\end{array}$ \\
\hline ZERO & $\begin{array}{c}\text {-ve } \\
\text { Sma } \\
11\end{array}$ & $\begin{array}{c}\text {-ve } \\
\text { Sma } \\
11\end{array}$ & $\mathrm{Z}$ & $\begin{array}{c}+ \text { ve } \\
\text { Smal } \\
1\end{array}$ & $\begin{array}{c}\text { +ve } \\
\text { Small }\end{array}$ \\
\hline NS & $\begin{array}{c}\text {-ve } \\
\text { Sma } \\
11\end{array}$ & $\mathrm{Z}$ & $\begin{array}{c}\text { +veS } \\
\text { mall }\end{array}$ & $\begin{array}{c}+ \text { ve } \\
\text { Smal } \\
1\end{array}$ & $\begin{array}{l}+ \text { ve } \\
\text { Big }\end{array}$ \\
\hline NB & $\mathrm{Z}$ & $\begin{array}{c}+\mathrm{ve} \\
\text { Sma } \\
11\end{array}$ & $\begin{array}{c}+ \text { ve } \\
\text { Smal } \\
1\end{array}$ & $\begin{array}{c}\text { +veB } \\
\text { ig }\end{array}$ & $\begin{array}{l}+ \text { ve } \\
\text { Big }\end{array}$ \\
\hline
\end{tabular}

The other groups have different colors. In such cases, the error as well as the error coefficient are considered to be differed by zeros as to make the regulator command fast and reduce the error. The rule has been formed as below: The first line and the first column consist of the linguistic terms with respect to the error as well as the error coefficient (+ve Big ... -ve Big). The intersection of online with one column describes the linguistic term corresponding to the output variable. Such representations of rules remain concise, compact, easy to read, and interpret. 


\section{Conclusion}

In this work FL based PD has been developed for water supply from and to the reservoir. It is implemented with an inexpensive 8-bit micro-controller and has been explained in the present work. Modifications have been made to make it smart and to meet the challenges for real time environment. The simulation shows better performances of the FL control as it is adaptable to the system. For future scope the rules may be optimized. Also the intelligent controller may be applied for other environment of the health care.

\section{REFFERENCES}

[1] Sarangi, L., Mohanty, M. N., \& Patnaik, S. (2017). Detection of abnormal cardiac condition using fuzzy inference system. International Journal of Automation and Control, 11(4), 372383.

[2] Lokanath, S., Narayan, M. M., \& Srikanta, P. (2016). Critical Heart Condition Analysis through Diagnostic Agent of e-Healthcare System using Spectral Domain Transform. Indian Journal of Science and Technology, 9(38).

[3] Sarangi, L., Mohanty, M. N., \& Patnaik, S. (2016). Design of ANFIS Based E-Health Care System for Cardio Vascular Disease Detection. In Recent Developments in Intelligent Systems and Interactive Applications (pp. 445-453). Springer International Publishing.

[4] Lokanath Sarangi, Mihir Narayan Mohanty, SrikantaPattnaik, "An Intelligent Decision Support System for Cardiac Disease Detection", IJCTA, International Press 2015.

[5] J. Yen, R. Langari: Fuzzy Logic: Intelligence, Control, and Information, Prentice-Hall, 1999.
[6] Y. S. Zhou, L. Y. Lai: Optimal Design for Fuzzy Controllers by Genetic Algorithms, IEEE Trans. On Industry Application, Vol. 36, No. 1, January/February 2000, pp. 93 - 97.

[7] Verbruggen, H. B. and Bruijn, P. M., 1997. Fuzzy control and conventional control: What is (And Can Be) the Real Contribution of Fuzzy Systems Fuzzy Sets Systems, Vol. 90, 151-160.

[8] Kowalska, T. O., Szabat, K. and Jaszczak, K., 2002. The Influence of Parameters and Structure of PI-Type Fuzzy-Logic Controller on DC Drive System Dynamics, Fuzzy Sets and Sysems, Vol. 131, 251-264.

[9] Ahmed, M. S., Bhatti, U. L., Al-Sunni, F. M. and El-Shafei, M., 2001. Design of a Fuzzy Servo-Controller, Fuzzy Sets and Systems, vol. 124: 231-247.

[10] Zilouchian, A., Juliano, M., Healy, T., 2000. Design of Fuzzy Logic Controller for a Jet Engine Fuel System, Control and Engineering Practices, Vol. 8: 873-883.

[11] Zadeh, L. A., 1965. Fuzzy sets, Information Control, Vol. 8, pp: 339353

[12] Liu, B. D., 1997. Design and Implementation of the Tree-Based Fuzzy Logic Controller, IEEE Transactions on Systems, Man, and Cybernetics, Part B: Cybernetics., Vol.27, No.3, 475-487.

[13] Zhiqiang, G., 2002. A Stable Self-Tuning Fuzzy Logic Control System for Industrial Temperature Regulation, IEEE 1886 Transactions on Industry Applications.Vol.38, No.2: 414-424.

[14] Shapiro, A. F., 2004. Fuzzy Logic in Insurance, Insurance: Mathematics and Economics, Vol.35, No.2, 399-424.

[15] Hayward, G. and Davidson, V., 2003. Fuzzy Logic Applications, Analyst, Vol.128, 1304-1306.

[16] Peri, V. M. and Simon, D., 2005. Fuzzy Logic Control for an Autonomous Robot, North American Fuzzy Information Processing Society, NAFIPS 2005 Annual Meeting, 337- 342.

[17] SofianeAchiche, Wang Wei, Zhun Fan and others 2007: Genetically generated double-level fuzzy controller with a fuzzy adjustment strategy. GECCO’07, July 7-11 